\title{
Distance graphs with maximum chromatic number
}

\author{
Javier Barajas $^{\dagger}$ and Oriol Serra ${ }^{\ddagger}$ \\ Universitat Politècnica de Catalunya \\ Jordi Girona, 1, E-08034 Barcelona, Spain \\ e-mail:jbarajas,oserra@mat.upc.es
}

Let $D$ be a finite set of integers. The distance graph $G(D)$ has the set of integers as vertices and two vertices at distance $d \in D$ are adjacent in $G(D)$. A conjecture of Xuding Zhu states that if the chromatic number of $G(D)$ achieves its maximum value $|D|+1$ then the graph has a clique of order $|D|$. We prove that the chromatic number of a distance graph with $D=\{a, b, c, d\}$ is five if and only if either $D=\{1,2,3,4 k\}$ or $D=\{a, b, a+b, a+2 b\}$ with $a \equiv 0 \quad(\bmod 2)$ and $b \equiv 1 \quad(\bmod 2)$. This confirms Zhu's conjecture for $|D|=4$.

Keywords: Distance graphs, chromatic number

\section{Introduction}

Let $X$ be a set and $\rho$ a distance in $X$. For a subset $D \subset \mathbb{R}$, the distance graph $G(D)=G(X, \rho, D)$ has vertex set $X$ and two vertices $x, y$ are adjacent in $G(D)$ if and only if $\rho(x, y) \in D$. Distance graphs on $X=\mathbb{R}$ and $X=\mathbb{Z}$ were first studied by Eggleton, Erdôs and Skilton $[4,5]$; when $X=\mathbb{Z}$ and $D$ is a finite set of integers, we have $\chi(D)=\chi(G(D)) \leq|D|+1$ see e.g.[10]. The determination of the chromatic number of distance graphs on the set of integers has been extensively treated in the literature, and several cases have been solved. From now on, unless explicitely stated otherwise, we will always consider distance graphs on the set integers with the usual distance. We also assume that $\operatorname{gcd}(D)=1$ since otherwise each connected component of $G(D)$ is isomorphic to $G\left(D^{\prime}\right)$ with $D^{\prime}=\{d / g, d \in D\}$ and $g=\operatorname{gcd}(D)$.

When $|D| \leq 2$ the chromatic number of $G(D)$ can be easily determined. If $|D|=1$ or all elements in $D$ are odd then $G(D)$ is bipartite. Otherwise, $\chi(D)=3$. After some partial results $[2,3,4,9]$ the case $|D|=3$ was finally settled by Xuding Zhu in [12], where sufficiently accurate bounds on the circular and fractional chromatic numbers of $G(D)$ are obtained to prove the following result.

\footnotetext{
${ }^{\dagger}$ Supported by the Ministry of Science and Technology of Spain, and the European Regional Development Fund (ERDF) under project-BFM-2002-00412 and by the Catalan Research Council under grant 2001SGR-00258.

$¥$ Supported by the Ministry of Science and Technology of Spain, and the European Regional Development Fund (ERDF) under project-BFM-2002-00412 and by the Catalan Research Council under grant 2001SGR-00258.
}

1365-8050 @ 2005 Discrete Mathematics and Theoretical Computer Science (DMTCS), Nancy, France 
Theorem 1 (Zhu,[12]) Let $D=\{a, b, c\}$ with $a<b<c$ and $\operatorname{gcd}(a, b, c)=1$. Then

$$
\chi(G(D))= \begin{cases}2, & \text { if } a, b, c \text { are all odd } ; \\ 4, & \text { if either } D=\{1,2,3 k\} \text { or } D=\{a, b, a+b\} \text { and } b-a \neq \equiv 0 \quad(\bmod 3) \\ 3, & \text { otherwise. }\end{cases}
$$

In this paper Zhu conjectured that, if the upper bound $\chi(G(D))=|D|+1$ is achieved then $D$ contains three not necessarily distinct elements $a, b, c$ with $a+b=c$. This conjecture was further strengthened to give the following one:

Conjecture 2 Let $D$ be a finite set of integers. If $\chi(G(D))=|D|+1$ then $D$ contains a clique of order $|D|$.

The distance graphs with clique number $\omega(G(D)) \geq|D|$ where characterized by Kemnitz and Marangio [7]. When $|D|=4$ they correspond to sets of the form $D=\{x, y, x+y, y-x\}, x<y$, or $D=\{x, 2 x, 3 x, y\}$, and the same authors determined the chromatic numbers of the corresponding distance graphs $[7,8]$.

For integers $x, n \in \mathbb{Z}$ we denote by $|x|_{n}$ the residue class of $x$ or $-x$ modulo $n$ in $\{0,1, \ldots,\lfloor n / 2\rfloor\}$. Let us denote by $G(D, n)$ the circulant graph $C a y\left(\mathbb{Z} / n \mathbb{Z}, D^{\prime} \cup-D^{\prime}\right)$, where $D^{\prime}=\left\{|d|_{n}, d \in D\right\}$. We also denote by $\chi(D, n)$ the chromatic number of $G(D, n)$. It was shown in [4] that a distance graph $G(D)$ always admit colouring with $\chi(D)$ colours which is periodic. Therefore, $\chi(D)$ is also the chromatic number of $G(D, n)$ for all multiples $n$ of the period of a periodic coloring of $G(D)$, that is,

$$
\chi(D)=\min \{\chi(D, n): n \in \mathbb{N}, n>\max \{D\}\} .
$$

This fact connects the problems of determining chromatic numbers of distance graphs and circulant graphs. The latter has been proved to be a NP-complete problem by Codenotti, Gerace and Vigna [1].

When $|D|=2$ the determination of the chromatic number of circulant graphs has been obtained by Yeh and Zhu [11] in the most interesting case when $D=\{1, x\}$ and in the general case, independently, by Heuberger [6]. This result provides a simple way to determine the chromatic number of the distance graph $G(D)$ when $|D|=3$ : given $D=\{a<b<c\}$ take, if possible, an appropriate value $N \in\{b+c, a+c\}$ to reduce the problem to the chromatic number of a circulant graph with two generators; then use the fact that $\chi(D) \leq \chi(D, N)$. This approach leaves only a few number of cases to deal with and leads easily to the determination of $\chi(D)$. This is the main idea used in this paper to settle Conjecture 2 for sets of four distances.

\section{The results}

In this context we prove the following result. For an integer $x \in \mathbb{Z}$, denote by $\nu_{p}(x)$ the $p$-adic valuation of $x$, that is, the largest power of prime $p$ dividing $x$.

Theorem 3 Let $p$ be a prime and $D$ a finite subset of integers. Let $m=\max _{d \in D} \nu_{p}(d)$.

$$
\text { If }\left|\nu_{p}^{-1}(i) \cap D\right| \leq 1 \text { for } i=0,1, \ldots, m \text { then } \chi(D) \leq\lceil 2 p /(p-1)\rceil \text {. }
$$

Proof: Take $N=p^{m+1}$ and consider $D^{\prime}=\left\{|d|_{N}, d \in D\right\}=\left\{d_{1}, d_{2}, \ldots, d_{k}\right\}$, where the elements in $D^{\prime}$ are ordered by decreasing values of $\nu_{p}$. Note that $0 \notin D^{\prime}$ and $\nu_{p}\left(|\lambda d|_{N}\right)=\nu_{p}(d)$ for each $d \in D$ and each $\lambda \in \mathbb{Z}_{N}^{*}$. 
We shall recursively define a sequence $u_{1}, \ldots, u_{k}$ of elements in $Z_{N}^{*}$ such that

$$
\left|\left(\prod_{i=1}^{k} u_{i}\right) d\right|_{N} \geq \frac{p-1}{2 p} N, \text { for each } d \in D
$$

The result then follows by Zhu's Lemma since

$$
\chi(D) \leq \chi_{N}(D)=\chi_{N}\left(D_{k}\right) \leq\lceil 2 p /(p-1)\rceil .
$$

Let $H$ be the subgroup of $\mathbb{Z}_{N}$ generated by $p^{m}$. Note that each coset of $H$ contains some element $x$ with $|x|_{N} \geq\left(\frac{p-1}{2}\right) p^{m}$.

We have $d_{1} \in H \backslash\{0\}$. Therefore there is $u \in\{1, \ldots, p-1\}$ such that

$$
\left|u d_{1}\right|_{N}=\frac{p-1}{2} p^{m}=\frac{p-1}{2 p} N .
$$

We define $u_{1}=u$.

Suppose that we have defined $u_{s} \in \mathbb{Z}_{N}^{*}$ for each $1 \leq s<i \leq k$ with the property that $\left|\left(\prod_{s<i} u_{s}\right) d_{j}\right|_{N} \geq$ $N(p-1) / 2 p$ for each $1 \leq j<i$. Let $v_{i-1}=\prod_{s<i} u_{s}$.

We have $v_{i-1} d_{i}=r_{i} p^{\nu_{p}\left(d_{i}\right)}$ for some $r_{i} \in \mathbb{Z}_{N}^{*}$. Then

$$
\left\{\left(r p^{m-\nu_{p}\left(a_{i}\right)}+1\right) v_{i-1} a_{i}, r=0,1, \ldots, p-1\right\}=\left\{r^{\prime} p^{m}+v_{i-1} a_{i}, r^{\prime}=0,1, \ldots, p-1\right\}=H+v_{i-1} a_{i} .
$$

Therefore there is $r \in\{0,1, \ldots, p-1\}$ such that $\left|\left(r p^{m-\nu_{p}\left(a_{i}\right)}+1\right) v_{i-1} a_{i}\right|_{N} \geq N(p-1) / 2 p$. Define $u_{i}=\left(r p^{m-\nu_{p}\left(a_{i}\right)}+1\right)$. Since $\nu_{p}\left(a_{j}\right)>\nu_{p}\left(a_{i}\right)$ for each $1 \leq j<i$, we have $v_{i} a_{j}=u_{i} v_{i-1} a_{j}=v_{i-1} a_{j}$. Hence,

$$
\left|v_{i} a_{j}\right|_{N} \geq N(p-1) / 2 p, j=1,2, \ldots, i .
$$

In this way we obtain the sequence $u_{1}, \ldots, u_{k}$ verifying (2).

When $p=2$ or 3 the following generalization holds.

Theorem 4 Let $m=m(p)$ denote the maximum value of $\nu_{p}(d), d \in D$. Then,

1. If $p=3$ and $\left|\nu_{p}^{-1}(i)\right| \leq 1$ for each $i<m$, then $\chi(D) \leq 3$.

2. If $p=2$ and $\left|\nu_{p}^{-1}(i)\right| \leq 1$ for each $i<m-1$, then $\chi(D) \leq 4$.

Theorems 3 and 4 are the essential tools we use to settle the first open case of Zhu's conjecture, when $|D|=4$. These theorems allow one to easily reduce the problem to the case in which $D=\{a, b, c, d\}$ verifies $\nu_{2}(a)=\nu_{2}(b)=0, \nu_{2}(c)=1$ and $\nu_{2}(d) \geq 2$. Some variations of the techniques used to prove Theorem 3 can then be applied leading to the following result.

Theorem 5 Let $D=\{a, b, c, d\}$ be a set of integers. If $\chi(D)=5$ then one of the following holds:

1. $D=\{1,2,3,4 n\}, n \in \mathbb{N}$.

2. $D=\{a, b, a+b, a+2 b\}$ with $a \equiv 0 \quad(\bmod 2)$ and,$b \equiv 1 \quad(\bmod 2)$. 
The main ideas behind the proof of Theorem 5 can be also applied to larger sets of distances. The following variation of Theorem 3 holds.

Theorem 6 Let $D$ be a set of distances. If $\left|\nu_{p}^{-1}(i) \cap D\right| \leq(p-1) / 2$ for each $i \in \mathbb{N}$ then $\chi(D) \leq p$.

Theorem 6 can be used to obtain some partial results to study Conjecture 2. For instance, for $|D|=5$ the following can be shown.

Proposition 7 Let $|D|=5$ and $\chi(D)=6$. Then $D$ contains an only multiple of 5 and there are elements $d, d^{\prime} \in D$ with $|d|_{5}=1$ and $\left|d^{\prime}\right|_{5}=2$.

\section{References}

[1] B. Codenotti, I. Gerace, S. Vigna, Hardness results and spectral techniques for combinatorial problems on circulant graphs. Linear Algebra Appl. 285 (1998), no. 1-3, 123-142.

[2] J. Chen, G. Chang and K. Huang, Integral distance graphs, J. Graph Theory 25 (1997), 281-287.

[3] W. Deuber and X. Zhu, The chromatic number of distance graphs Discrete Math. 165/166 (1997), 195-204.

[4] R.B. Eggleton, P. Erdôs and D.K. Skilton, Colouring the real line, J. Combinatorial Theory Ser. B 39 (1985) 86-100.

[5] R.B. Eggleton, P. Erdős and D.K. Skilton, Coluring prime distance graphs, Graphs and Combinatorics 6(1990) $17-32$

[6] C. Heuberger, On planarity and colorability of circulant graphs, Discrete Math. 268 (2003) 153-169.

[7] A. Kemnitz and M. Marangio, Colorings and list colorings of integer distance graphs, preprint 2003

[8] A. Kemnitz and M. Marangio, Chromatic number of integer distance graphs,Discrete Math. 233 (2001), 239-246.

[9] M. Voigt, Coloring of Distance graphs, Ars Combinatoria 52 (1999) 3-12.

[10] H. Walther, Über eine spezielle Klasse unendlicher Graphen Graphentheorie, Bd.2. Bibl. Inst., Mannheim (1990) 268-295.

[11] H.G. Yeh and X. Zhu, 4-colourable 6-regular toroidal graphs, Discrete Math. 273 (2003) 261-274.

[12] X. Zhu, Circular chromatic number of distance graphs with distance sets of cardinality three. $J$. Graph Theory 41 (2002), no. 3, 195-207. 